\title{
Commentary
}

\section{Herd immunity: basic concepts, epidemiological consideration and its application during pandemic like COVID-19}

\author{
Smita Sinha ${ }^{1 *}$, Rishita Chandra ${ }^{2}$ \\ ${ }^{1}$ Department of Community and Family Medicine, ${ }^{2}$ School of Public Health, All India Institute of Medical Sciences,
} Rishikesh, Uttarakhand, India

Received: 24 July 2020

Accepted: 04 September 2020

*Correspondence:

Dr. Smita Sinha,

E-mail: smita.mailid@gmail.com

Copyright: (C) the author(s), publisher and licensee Medip Academy. This is an open-access article distributed under the terms of the Creative Commons Attribution Non-Commercial License, which permits unrestricted non-commercial use, distribution, and reproduction in any medium, provided the original work is properly cited.

\section{INTRODUCTION}

The meaning of the term "Herd immunity" or "Herd protection" can be understood as "when most of the population is immune to an infectious disease, this provides indirect protection to rest who are not immune to the disease".

Innate immunity is inherited and is present in one's body since birth. When it gets activated against a specific type of antigen, the immunity remains throughout life. Acquired immunity is established at the level of the individual, when there is a natural infection with a pathogen or via immunization. Adaptive or acquired immunity is created in response to exposure to a foreign substance.

"Herd immunity is referred as the indirect protection from infection caused to susceptible individuals when a sufficiently large population of immune individuals exist in a population. It is derived from the effects of individual immunity brought to the level of the population. It refers to the indirect protection from infection conferred to susceptible individuals when a sufficiently large proportion of immune individuals exist in a population". ${ }^{1}$

For example, if $80 \%$ of the population gets immune to a specific virus, four out of every five people if come in contact with the disease will neither be affected nor spread it further and hence the disease transmission would stop in the population.
The proportion of the population needed to get herd immunity depends on how contagious the infection is, generally, in order to achieve herd immunity, $70 \%$ to $90 \%$ individuals need to be immune.

The effect at the level of population is often considered in relation to immunization programs. The main aim of such vaccination programs is establishing herd immunity. The individuals who are left or not vaccinated are still protected against disease as the number of susceptible hosts would be less.

\section{TRANSMISSION DYNAMICS AND HERD IMMUNITY}

An essential of disease control in case of infectious diseases is determination of the route of transmission and to break the transmission chain. The concept of transmission doesn't only comprise of the spreading but it consists of the pathogen fitness, presentation of the pathogen by a host, pathogen movement from infected to healthy person, and targeting new host.

Transmission being a complex process can be explained through simplified models by, one parameter, $\beta$, i.e. transmission coefficient. The complexity of $\beta$, can be sublimed by considering 'the force of infection' $F$, that denotes the rate at which healthy persons are getting infected. It depends on the number infected and susceptible hosts, I and S respectively. ${ }^{2}$

The point at which the proportion of susceptible individuals falls below the threshold needed for 
transmission is known as the herd immunity threshold. Herd immunity comes to effect at this level benefitting the susceptible people by indirect protection from infection.

The threshold of herd immunity depends on basic reproduction number, a parameter denoted as $R_{0}$. $R$ naught is an indicator of the contagiousness or transmissibility of infectious and parasitic agents. The basic reproduction number indicates the risk of an infectious agent in close proximity to the spread of an epidemic. $\mathrm{R}_{0}$ refers to the average number of secondary infections caused by a single infectious individual into a completely susceptible population. ${ }^{3}$ The term basic reproduction number $R_{0}$ denotes the number of new infections that can be spread by infected individuals to non-immune and susceptible contacts during the period of infectivity. It indicates the transmissibility of a virus.

Let us assume a pathogen having R0 of four. This suggests that in a hypothetical situation with no existing immunity in the population, four new individuals can encounter the disease by one infected host. In Mathematical expression, the herd immunity threshold is defined by $1-1 / \mathrm{R}$ " (for example if $\mathrm{R}_{0}=4$, the threshold for herd immunity comes out to be 0.75$){ }^{3}$

Therefore, $\mathrm{R}_{0}$ would be greater for the pathogens with higher communicability and the proportion of the population needed to be immune to block the chain of transmission will also be greater. For $\mathrm{R}_{0}>1$, the number of infected will increase, and for $\mathrm{R}_{0}<1$, the transmission can become extinct.

Another significant parameter in this context is the effective reproduction number, $\mathrm{R}_{\mathrm{e}}$. Re is defined as the average number of secondary cases generated by a single index case over an infectious period in a partially immune population. $^{4}$

$\mathrm{R}_{\mathrm{e}}$ and $\mathrm{R}_{\mathrm{o}}$ are different from each other. The dynamics of this parameter is likely to change upon unfolding some vaccination campaign. The vaccination programs aim to achieve $R_{e}$ below 1 . This happens when the immune proportion of individuals in the population exceeds the herd immunity threshold. The pathogen spread is interrupted and the number of infections decline. ${ }^{3}$

\section{HERD IMMUNITY, HISTORY AND EVOLUTION}

A study conducted by Topley and Wilson about a century ago mentioned bacterial infection and herd immunity. They suggested the need for a careful study about the factors that determine the immunity of a herd as distinguished from the immunity of an individual. ${ }^{5}$

In the year 1970, Smith recognized simple threshold theorem, which states that if random delivery of immunity i.e. via vaccination is done on a randomly mixed population such that on an average each individual could contact R0 individuals in a manner sufficient for transmission, then the incidence of the infection would decline if the proportion of immune exceeded $\left(\mathrm{R}_{0}-1\right) / \mathrm{R}_{0}$, or $1-1 / R_{0}$. This theorem happens to be a milestone in the history of the concepts of herd immunity. ${ }^{6}$

Though other studies considered this highly theoretical. Various arguments had been given and they emphasized on the importance of heterogeneity in a population. Therefore, the concept of simple thresholds was not considered apt from the aspect of public health considering the assumptions of homogeneous mixing. In spite of considering herd immunity as a threshold, it needs to be taken as a continuum.

\section{EVIDENCE BASED HERD IMMUNITY FACTS AND EXAMPLES}

The concept of vaccination or immunization programs and herd immunity go hand in hand. The most effective examples have been achieved through various vaccination programs. The theory behind vaccines and herd immunity states that by inducing immunity against infection, in a randomly mixed population which is consistent with the threshold for herd immunity can be represented as $\mathrm{V}_{\mathrm{c}}=\left(1-1 / \mathrm{R}_{0}\right)$, where $\mathrm{Vc}$ is the critical minimum proportion to be vaccinated. $^{7}$

This concept of herd immunity reflects that when a large proportion of the population is vaccinated or immunized, can prevent outbreaks can be prevented by protecting both unvaccinated as well as vaccinated individuals. The rate of reduction in transmission via vaccination in a population depends upon various factors, i.e. pathogenicity of the agent, its transmissibility, the web of human interactions, frequency of contacts, and also on vaccine distribution and its effectiveness. For example, selective vaccination program against influenza in school children of Japan during the 1990s had shown reduction in morbidity and mortality amongst the elderly group of individuals. ${ }^{8}$

Other milestones achieved are the references from wideranging childhood infections like measles, mumps, rubella, polio, pertussis and chickenpox. They have been a game-changer as the critical number of susceptible individuals was higher. The vaccination program helped to keep the number of susceptible individuals below the critical number in order to prevent an epidemic. ${ }^{9,10}$

Some commendable examples of indirect protection came into existence with the introduction of conjugate vaccines for the infection caused by Haemophilus and Pneumococcus. The incidence of disease were reduced among the population as conjugate vaccines provided protection against disease as well as infectiousness too. ${ }^{11}$

Referring to vaccine-based eradication programs globally, success achieved in the case of smallpox and polio has been impressive. India was declared polio-free by 2014 
and successfully certified the criteria of getting herd immunity. ${ }^{12}$ importantly; the indirect protection is an outcome of the vaccine's effectiveness. The vaccines alter the infectiousness along with the prevention of disease. In the absence of this phenomenon, indirect immunity cannot be achieved and so there would be no herd immunity. But if the vaccine coverage is low, the program may fail to achieve herd immunity, as sometimes outbreak in vaccine-preventable diseases are seen. E.g. Measles outbreak in 2019. ${ }^{13}$ Also, immunity for various other infections is prone to waning example pertussis, rotavirus etc. promoting periodic outbreaks.

\section{HERD IMMUNITY AND COVID-19 PANDEMIC}

The global pandemic of COVID-19 has caused about 6,931,000 clinically confirmed cases and has reached the death toll of more than 400,000 globally as of June 8, 2020. Currently, the trials for vaccines and drugs for the prevention and treatment of SARS-CoV-2 infection is going on. The outcomes of these trials are eagerly awaited but the effectiveness of the interventions are still not known with an estimated period of one to one and a half years of waiting. In the absence of any vaccine, establishing herd immunity for SARS-CoV-2 via natural infection happens to be just a theoretical equation.

The transmission dynamics of SARS-CoV-2 is still not clear. The exponential growth rate of the outbreak was estimated to be $0.1-0.14 /$ day initially. The $\mathrm{R}_{0}$ estimated from 425 cases in the province of Wuhan, China, was 2.2 i.e. on an average, 2.2 new infections can be caused by each infected individual. ${ }^{14}$ Recently, the R0 has been estimated to be higher i.e. 5.7, most of the estimates lie in this range. ${ }^{15}$

There are numerous complexities in understanding the dynamics of COVID-19 spread. SARS-CoV-2 being a novel virus and showing variation in $\mathrm{R} 0$ across different populations with inter-individual variation, demography and time make the estimation of community spread as well as epidemic decline more difficult given the limited data available. The pandemic and post-pandemic The transmission dynamics of SARS-CoV-2 not only depends on the immunity threshold, but also on its duration as well as cross-immunity between the novel coronavirus and other coronaviruses and also on the measures to control and contain the disease. The seasonal variation, ethnicity, age and sex specific variation in the case fatality rates have been reported which signifies the differences in susceptibility as well as complications related to COVID19 disease.

In such a scenario throwing a concept like Herd immunity, when epidemiology of a novel virus is still not well understood and requires further research to explore more, might have devastating results in the community.

\section{CAN HERD IMMUNITY THRESHOLD BE REACHED FOR SARS-COV-2 IN THE ABSENCE OF A VACCINE?}

The relationship between the concept of herd immunity and SAR-CoV-2 infections can be projected to numerous assumptions, e.g. homogeneity in the population, lifelong immunity etc. Inability of an asymptomatic individual to spread the disease is yet not established. The asymptomatic carriers can be highly infectious and can increase the transmission significantly; hence clinical manifestation cannot be a good indicator of transmissibility in this case. Also, the CFR of COVID-19 is non-uniform across different age groups, with the highest number of deaths among individuals of 60 years or more. SARS-CoV-2 is a novel pathogen, many features of its transmission and infection dynamics could not be characterized well, making it difficult to comment over its herd immunity threshold.

Herd immunity is highly effective for pathogens in which sterilizing or lifelong immunity can be induced. The efficacy of herd immunity is mainly dependent on the strength and duration for which a population can stay immune. The ability of SARS-CoV-2 to establish herd immunity articulates on the assumption of it being sufficient to induce protective immunity. Presently, the extent to which the population can develop lifelong immunity to SARS-Cov-2 is not clear. Hence, the group of susceptible hosts that can contact each other frequently due to uneven and unclear characteristics of immunity can become the pocket of risk for local outbreaks.

With massive sample testing and individuals getting rapid tests done, it is very important to assure the authenticity of the test i.e. the negative cases should be truly negative. Another dimension that needs to be addressed is the reactivation of previously infected cases, recovered and those released from isolation. Bao et al conducted a study among Rhesus macaques for assessing the chances of SARS-CoV-2 reinfection. The result did not show any evidence of reinfection occurring after one month of the first viral challenge. Hence, it was concluded that these animals have sterilizing immunity, though for a short term. ${ }^{16}$ A study was done by $\mathrm{Wu}$ et al in a group of 175 recovered COVID-19 patients. A considerable titer of SARS-CoV-2-specific serum neutralizing antibodies (NAbs) were detected in 165 individuals. ${ }^{17}$ These findings might instil optimism towards breaking the chain of transmission through herd immunity, but the questions that arise at this point of time is reliability on NAb titers and the duration of acquired immunity.

According to a report, in South Korea, 116 COVID-19 recovered individuals $\mathrm{w}$ again came positive. ${ }^{18}$ Korea followed an approach to mark a patient as negative by considering two consecutive samples within the period of 24 hours. This brings our attention to the necessity of more precautions to be sure a patient has truly recovered from COVID-19 or may not reactivate again after 
recovery. The confirmed cases of SARS have demonstrated that the responses from the neutralizing antibody (Nab) could last from months to two years, and the decrease in titers is observed by about 15 months. ${ }^{19}$ So, in order to get the herd immunity for 60 $70 \%$ of the population, there is a need for mass serological testing to determine the number of infected and immune persons. This might help to draw an estimate for reaching the herd immunity threshold.

SARS-CoV-2 and SARS-CoV viruses share a close relationship in their microbiology. The RNA genomes of the viruses are about $80 \%$ identical. During the SARS outbreak in 2003, the concept of herd immunity remained unclear along with the host response in viral clearance or tissue damage. The initial viral load had been high and was considered to be independently associated with the disease severity and host immune response. The family of RNA coronaviruses are found to have genome instability, so the risk of SARS-CoV is still thought to be high hence nullifying the theory of achieving herd immunity without immunization. ${ }^{20}$

In such a situation, where the basic facts of epidemiology i.e. from $\mathrm{R}_{0}$, basic reproduction number of the virus to waning of serological antibodies, reinfection and reactivation of the virus, everything needs further research and evidence.

The idea of establishment of herd immunity to SARS$\mathrm{CoV}-2$, in absence of any vaccine is going to overwhelm already torn and burdened healthcare infrastructure during a pandemic by allowing a large proportion of the population to encounter the infection deliberately. This effect tends to have devastating effect on countries with limited hospital capacity. It can dilapidate the already not so robust public health infrastructure.

\section{CONCLUSION}

Herd immunity is intended to provide indirect protection to susceptible individuals by minimizing the chances of effective contact and comes to effect when the threshold of herd immunity is achieved in a population. In case of a novel pathogen e.g. SARS-CoV-2, whose epidemiology is complex and still under evolution with no availability of vaccines, attaining herd immunity should not be the ultimate goal. Rather, guidelines and policies to contain the disease transmission and vulnerable groups should be given emphasis. After an effective vaccine for SARSCoV-2 is approved, the phenomenon of herd immunity might be taken as a primary objective and can be achieved through global immunization programs with high vaccine coverage and strict surveillance. But, at present, the concept of herd immunity in relation to COVID-19 appears more to be theoretical which might have detrimental effects.

\section{REFERENCES}

1. Randolph HE, Barreiro LB. Herd immunity: understanding COVID-19. Immunity. 2020;52:73741.

2. Antonovics J. Transmission dynamics: critical questions and challenges. Phil Trans R Soc B. 2017; 372:20160087.

3. Anderson RM, May RM. Vaccination and herd immunity to infectious diseases. Nature. 28;318:3239.

4. Delamater PL, Street EJ, Leslie TF, Yang YT, Jacobsen KH. Complexity of the Basic Reproduction Number $\left(\mathrm{R}_{0}\right)$. Emerging Infectious Diseases. 2019; 25:1-4.

5. Topley WWC, Wilson GS. The Spread of Bacterial Infection. The Problem of Herd-Immunity. The Journal of Hygiene. 1923;21:243-9.

6. Smith CEG. Prospects for the Control of Infectious Disease. Proceedings of the Royal Society of Medicine. 1970;63:1181-90.

7. Vynnycky E, White R. An Introduction to infectious disease modelling. Oxford University Press. 2010; 400.

8. Reichert TA, Sugaya N, Fedson DS, Glezen WP, Simonsen L, Tashiro M. The Japanese experience with vaccinating schoolchildren against influenza. New England Journal of Medicine. 2001;344:889-96.

9. Hedrich AW. Monthly estimates of the child population susceptible to measles. American Journal of Epidemiology. 1933;17:613-36.

10. Hamer WH. Epidemic disease in England - the evidence of variability and of persistency of type. The Lancet.1906;1:733-9.

11. American public health association, control of communicable diseases manual. Available at https://www.apha.org/ccdm. Accessed on 5 June 2020.

12. John TJ, Vashishtha VM. Eradicating poliomyelitis: India's journey from hyperendemic to polio-free status. Indian Journal of Medical Research. 2013; 137:881-94.

13. 13.World health organization| measles- global situation. Available at: https://www.who.int/ immunization/diseases/measles/en/. Accessed 5 June 2020.

14. Li Q, Guan X, Wu P, Wang X, Zhou L, Tong Y, et al. Early Transmission Dynamics in Wuhan, China, of Novel Coronavirus-Infected Pneumonia. New England Journal of Medicine. 2020;382:1199-207.

15. Sanche S, Lin YT, Xu C, Romero-Severson E, Hengartner N, Ke R. High contagiousness and rapid spread of severe acute respiratory syndrome coronavirus 2. Emerging Infectious Diseases. 2020; 26.

16. Bao L, Deng W, Gao H, Xiao C, Liu J, Xue J, et al. Reinfection could not occur in SARS-CoV-2 infected rhesus macaques. BioRxiv. 2020;3(13):1-17.

17. Wu F, Wang A, Liu M, Wang Q, Chen J, Xia S, et al. Neutralizing antibody responses to SARS-CoV-2 in a 
COVID-19 recovered patient cohort and their implications. MedRxiv. 2020;3(30):1-10.

18. Smith J. South Korea reports more recovered coronavirus patients testing positive again. Available at: https://www.reuters.com/article/us-healthcoronavirus-southkorea-idUSKCN21S15X. Accessed 5 June 2020

19. Mo H, Zeng G, Ren X, Li H, Ke C, Tan Y, et al. Longitudinal profile of antibodies against SARScoronavirus in SARS patients and their clinical significance. Respirology. 2006;11:49-53.
20. $\mathrm{Li} \mathrm{CK}, \mathrm{Xu} \mathrm{X}$. Host immune responses to SARS Coronavirus in humans. Molecular Biology of the SARS-Coronavirus. 2009;259-78.

Cite this article as: Sinha S, Chandra R. Herd Immunity: basic concepts, epidemiological consideration and its application during pandemic like COVID-19. Int J Community Med Public Health 2020;7:4216-20. 